ÉGYPTE monde arabe

\section{Égypte/Monde arabe}

$12 \mid 2015$

Evolution des systèmes médiatiques après les révoltes arabes

\title{
Al-Badîl, ou L'alternative. Récit d'une expérience à la croisée entre journalisme et engagement militant
}

\section{Marianna Ghiglia}

\section{(2) OpenEdition}

\section{Journals}

Édition électronique

URL : https://journals.openedition.org/ema/3428

DOI : $10.4000 /$ ema.3428

ISSN : 2090-7273

Éditeur

CEDEJ - Centre d'études et de documentation économiques juridiques et sociales

Édition imprimée

Date de publication : 25 mars 2015

Pagination : 115-145

ISBN : 9782905838858

ISSN : 1110-5097

Référence électronique

Marianna Ghiglia, «Al-Badîl, ou L'alternative. Récit d'une expérience à la croisée entre journalisme et engagement militant », Égypte/Monde arabe [En ligne], 12 | 2015, mis en ligne le 25 mars 2015, consulté le 07 juillet 2022. URL : http://journals.openedition.org/ema/3428 ; DOI : https://doi.org/ 10.4000/ema.3428 


\section{Marianna Ghiglia}

\section{RÉSUMÉ / ABSTRACT}

Le processus de démonopolisation de l'espace médiatique égyptien en cours depuis la fin des années 1990 se caractérise, entre autres, par l'émergence d'une presse privée qui se dit indépendante à la fois de l'Etat et du champ politique traditionnel. Bien que son impact sur I'espace public national ait été vite perçu par nombre d'observateurs, ce phénomène n'a pas fait l'objet d'études particulières. Cet article se propose de retracer l'histoire d'un journal privé 'indépendant' qui paraît sur la scène médiatique en juillet 2007 : le quotidien Al-Badîl (L'Alternative). Saisie dans une perspective diachronique, et par les acteurs individuels et collectifs dont elle est à chaque fois le produit, I'expérience d'Al-Badîl révèle une profonde imbrication entre les univers du journalisme et de l'engagement militant. Le journal est un lieu de confrontation symbolique entre deux manières différentes de concevoir le journalisme et l'engagement militant et un espace où s'opère le passage d'une génération intellectuelle et militante à une autre.

\section{MOTS CLÉS :}

Presse privée, médias, générations, profession journalistique, engagement militant .

The Egyptian media system has been affected by a clear process of de-monopolization since the late 1990's. This led to the appearance, among other things, of a new private press that claimed to be independent from both the State and traditional political field. Although its impact on the national public sphere was quickly perceived as relevant by many observers, this phenomenon in itself has not been studied. This article seeks to trace the history of an 'independent' private newspaper that first appeared on the media scene in July 2007: the daily paper Al-Badîl (The Alternative). Studied in a diachronic perspective and through its individual and collective actors, Al-Badîl's 'experiment' reveals a deep overlap between the worlds of journalism and political activism. The newspaper is a space of symbolic confrontation between two different ways of perceiving journalism and political activism and the site of a switch process from an intellectual and political generation to another. 


\section{KEYWORDS:}

Private press, Mass media, Generations, Journalistic profession, Political activism.

MARIANNA GHIGLIA est doctorante allocataire de recherche à I'AixMarseille Université / IREMAM, où elle prépare une thèse en histoire du temps présent et sociologie politique sous la co-direction de Ghislaine Alleaume et Myriam Catusse. Son travail de recherche porte sur la presse privée en Egypte et son impact sur la reconfiguration à la fois de l'espace public national et de la profession journalistique.

Courriel : marianna.ghiglia@gmail.com

MARIANNA GHIGLIA is a PhD research fellow at Aix-Marseille University / IREMAM, where she is preparing a thesis in Contemporary History and Political Sociology under the joint supervision of Ghislaine Alleaume and Myriam Catusse. Her research focuses on the private press in Egypt and its impact on the reconfiguration of both the national public sphere and the journalistic profession.

Email: marianna.ghiglia@gmail.com 


\section{AL-BADÎL, OU L'ALTERNATIVE RÉCIT D’UNE EXPÉRIENCE À LA GROISÉE ENTRE JOURNALISME ET ENGAGEMENT MILITANT ${ }^{1}$}

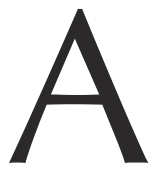

u cours des dernières décennies, le paysage médiatique égyptien a subi une profonde reconfiguration. L'avènement des nouvelles technologies de l'information et de la communication, dont les chaînes satellitaires transnationales et l'internet, a entraîné une progressive perte de contrôle, de la part de l'Etat autoritaire, sur les flux informationnels traversant le pays. Dans ce contexte, les autorités égyptiennes n'ont pas essayé de mettre un frein à un phénomène qu'elles ne pouvaient de facto pas contrôler, mais elles se sont vues contraintes d'intervenir pour mettre en œuvre une libéralisation contrôlée du secteur de I'information et des médias traditionnels ${ }^{2}$. Démonopolisation maîtrisée de l'espace télévisuel, car les licences pour la création de chaînes privées égyptiennes ne sont accordées qu'à des hommes d'affaires considérés comme proches du régime. Démonopolisation moins contrôlée en matière de presse écrite, ce qui permet la prolifération de nombreux journaux privés. Or, cette libéralisation du champ médiatique est d'une certaine manière "contrebalancée» par le verrouillage progressif des institutions politiques, syndicales et associatives du pays, phénomène qui a été mis en lumière par certains chercheurs ${ }^{3}$. Ainsi, face à la fermeture des instances traditionnelles de la représentation politique, I'espace médiatique se transforme en quelque sorte en

1. Cet article est partiellement tiré d'un mémoire de Master 2 intitulé "Histoire d'un journal égyptien " alternatif»: I'expérience du quotidien Al-Badîl ", préparé sous la direction de Ghislaine Alleaume et soutenu en septembre 2011 à l'Université de Provence.

2. El-Khawaga, 2003 (b).

3. Kienle, 2001 ; Ben Néfissa, 2010. 
une arène d'expression du politique et est graduellement investi par l'opposition égyptienne. Alors que la jeunesse éduquée du pays fait du Web sa sphère privilégiée d'expression de griefs et de critique de l'autorité, en investissant la blogosphère d'abord et ensuite les réseaux sociaux, les élites intellectuelles et journalistiques se focalisent sur des supports médiatiques plus traditionnels, notamment la presse. Les journaux privés dits 'indépendants' qui émergent au cours des années 2000 deviennent le canal d'expression d'une partie de l'opposition égyptienne et contribuent de manière évidente à élargir les limites du 'dicible' dans l'espace public du pays en brisant les tabous les plus solides, dont la critique de la figure du Président. Si certains spécialistes ont perçu l'importance du rôle joué par la presse indépendante et ont avancé l'hypothèse qu'elle aurait fourni, entre autres, une sorte de protection aux acteurs des mobilisations sociales de la période 2006 - 2010 en 'publicisant' leurs actions collectives ${ }^{4}$, ces publications n'ont pas fait l'objet d'études particulières et la question des acteurs qui leur donnent naissance et qui les font vivre - groupes hétérogènes composés la fois d'hommes d'affaires et de journalistes / intellectuels - a été négligée.

Cet article se propose de retracer l'histoire singulière d'un des représentants de la presse indépendante égyptienne, le quotidien AlBadîl (L'Alternative). Expérience à la fois originale et épineuse : paru pour la première fois en juillet 2007 grâce à l'initiative d'un groupe de militants de gauche, contraint à la fermeture en avril 2009 officiellement pour des raisons financières, il sera relancé en novembre 2010 sous un nouveau nom et un nouveau format par une partie des journalistes qui y travaillaient. Saisie dans une perspective diachronique, et par les acteurs individuels et collectifs dont elle est à chaque fois le produit, I'expérience d'Al-Badîl révèle une profonde imbrication entre les univers du journalisme et de l'engagement militant. Pour être plus précis, le journal est un lieu de confrontation symbolique entre deux manières différentes de concevoir le journalisme et l'engagement militant et un espace où s'opère le passage d'une génération intellectuelle et militante à une autre.

4. Bernard-Maugiron, 2007. 


\section{ENTRE GAUCHE DÉMOCRATIQUE ET GÉNÉRATION '70 : «FONDER LA TROISIÈME VOIE ...»}

Le 16 juillet 2007 paraît sur la scène journalistique égyptienne un nouveau quotidien privé ${ }^{\text {: }}$ Al-Badîl. Le nom du journal révèle immédiatement l'intention des acteurs qui ont contribué à le faire naître : proposer une voix alternative à celles existantes. En s'autodéfinissant comme "indépendant" (sahîfa yaumiya mustaqilla), le quotidien se place dès le début en opposition directe vis-à-vis des médias de l'Etat, critiqués pour leur manque de professionnalisme. Alors que ces derniers méprisent le public en le considérant comme un " 'troupeau ensorcelé' ou susceptible de se faire ensorceler ", "AlBadîl appartient à une école complètement opposée, selon laquelle la mission de la presse est de fournir des informations correctes, présenter des opinions et des propositions éclairantes et élargir le champ des choix possibles devant une opinion publique avertie $»^{6}$. Un éditorial publié à la Une clarifie d'emblée les objectifs envisagés par le journal : celui-ci «se propose d'éclairer et promouvoir la lutte pour la démocratie et les libertés publiques et de garantir la réalisation des droits économiques et sociaux de tous les Egyptiens sur un pied d'égalité ${ }^{7}$. Si ces mots montrent à l'évidence qu'Al-Badîl ne se contente pas d'être un simple témoin de l'actualité, mais revendique un statut $\mathrm{d}^{\prime}$ " acteur", un deuxième article éclaircit davantage la mission qu'il s'attribue: "L'alternative ?! Oui, I'alternative qui est devenue une nécessité urgente, l'alternative qui est devenue un objectif et un rêve pour faire face à une réalité intolérable, l'alternative pour s'opposer à la corruption, à la dictature, à la domination et à l'exploitation, l'alternative pour un monde plus juste, l'alternative que nous souhaitons et convoitons, $c^{\prime}$ est l'alternative construite par les gens eux-mêmes pour réaliser leurs intérêts et objectifs. Voici entre les mains du lecteur le quotidien L'alternative, qui se considère comme étant une partie vivante et active de ce mouvement, reflète ses objectifs et aspirations et ambitionne même de contribuer à les réaliser $»^{8}$. Au-delà de ses objectifs déclarés et des modalités discursives de présentation de soi, quelles sont les origines de ce projet éditorial ? Qu'en est-il des fondateurs et pourquoi ont-ils décidé d'investir l'espace public du pays en créant une nouvelle publication?

5. C'est le troisième quotidien "indépendant" à faire son entrée dans l'espace journalistique égyptien, après Al-Masrî Al-Yaum (2004), et Al-Dustûr (paru en 2005 en tant qu'hebdomadaire, son édition quotidienne sort en mars 2007).

6. "Le peuple est I'alternative", Al-Badîl, 16 juillet 2007, p. 1.

7. Ibid.

8. "Ensemble nous construisons I'alternative", Al-Badîl, 16 juillet 2007, p. 2. 
Contrairement à la plupart des journaux privés qui voient le jour dans cette période d'effervescence sociale et politique, Al-Badîl n'est pas le fruit du mariage entre journalistes et capital ${ }^{9}$. C'est le produit $d^{\prime} u n e$ initiative collective lancée par un groupe d'intellectuels et militants de la gauche égyptienne, dont plusieurs activistes des droits de l'homme.

A en croire les acteurs ayant participé au projet depuis la phase initiale, l'idée de la publication serait née au sein du Centre Egyptien Social Démocrate (al-markaz al-misrî al-ijtimâî al-dîmûqrâtî), entité politique informelle dont la création remonte à 2002 et qui rassemble quelques dizaines de militants d'âges variés. Ayant été socialisés, très jeunes, dans des organisations marxistes clandestines, ces derniers sont tous en rupture avec leurs précédentes expériences politiques. Ils critiquent d'une part l'excessive centralisation et l'autoritarisme du fonctionnement interne des partis de gauche et d'autre part, la place prépondérante qu'occupent le référent nationaliste et les slogans patriotiques dans leurs systèmes doctrinaux ${ }^{10}$, ce qui aurait en quelque sorte dénaturé l'esprit marxiste ${ }^{11}$. Cette remise en question débouche sur l'élaboration d'un nouveau projet idéologique désigné sous le nom de "gauche démocratique " et fondé sur la jonction " entre les principes de la démocratie et des droits de l'homme, d'un côté, et celui de la justice sociale, de l'autre ${ }^{12}$, pour reprendre les mots de Farid Zahran, un des promoteurs du centre. L'idée de publier un journal aurait éclos face aux difficultés de se constituer en parti et $d^{\prime}$ investir la scène politique légale du pays. Comme l'explique très clairement un de ces activistes, " nous avions l'ambition de fonder un parti, mais nous n'avons jamais réussi à concrétiser notre objectif. Fonder un parti en Egypte est presque impossible, non seulement pour des raisons de nature juridique, mais aussi parce qu'il est difficile de rassembler un nombre suffisant de militants et de cadres. Ainsi, nous avons décidé de créer un journal $»^{13}$. Al-Badîl aurait donc à l'origine été conçu comme un instrument d'action politique de «substitution ». L'accès à la rue

9. Le phénomène par lequel des journalistes égyptiens désireux de créer de nouvelles publications ont commencé à chercher des mécènes pour financer leurs initiatives remonte aux années 1990. Voir : El-Khawaga, 2000.

10. Comme le montre l'étude de Gennaro Gervasio sur la mouvance communiste égyptienne des années 1970 (Gervasio, 2010), dès la fin des années 1940 le thème de la « question nationale » a pris une centralité remarquable dans le discours politique de toutes les formations marxistes au détriment de la « question sociale ». La tendance « nationaliste » de la gauche égyptienne s'est ensuite ultérieurement exacerbée dans les années 1970 sous le coup de la défaite de juin 1967 et de l'occupation du Sinaï par I'armée israélienne.

11. Entretiens avec plusieurs activistes du CESD.

12. Entretien personnel, Le Caire, 13 avril 2011.

13. Entretien personnel avec SS, Le Caire, 27 juillet 2010. 
étant de fait interdit à l'opposition égyptienne dans son ensemble, il s'agit d'investir l'espace public en passant par le champ médiatique ${ }^{14}$. Soulignons que certains militants du CESD ont déjà lancé, en juin 2005, une revue à parution irrégulière intitulée $\mathrm{Al}$-Busla $(\text { La Boussole })^{15}$. Imprimée à 1000 exemplaires et consacrée à la publication de longs articles d'analyse politique et sociale, elle est par définition destinée à un public d'élite, alors qu'un journal d'information générale offre l'opportunité de s'adresser à un lectorat plus large, voire même de viser les masses.

Si l'idée initiale est le fait d'un collectif précis, la naissance d'AlBadîl doit beaucoup à la participation d'autres acteurs qui ne font pas strictement partie de ce groupe. L'initiative prend de l'essor grâce à une figure intellectuelle renommée de la gauche égyptienne : Mohammed Al-Sayyed Saïd. Impliqué dans le projet dès la première heure, ce dernier est considéré comme étant le père spirituel du journal et deviendra également son premier rédacteur-en-chef. Né en 1950, diplômé de la Faculté d'Economie et de Sciences Politiques de l'Université du Caire, Mohammed Al-Sayyed Saïd est vice-directeur du Centre des études politiques et stratégiques d'Al-Ahrâm, I'une des plus importantes institutions publiques de recherche. Cela ne l'empêche pas d'être parmi les esprits les plus critiques vis-à-vis des autorités au pouvoir. En 2004, il fait partie du groupe d'intellectuels à l'origine du « Mouvement égyptien pour le changement ». Plusieurs observateurs ont par ailleurs remarqué le discours qu'il prononce en février 2005 à la Foire du livre du Caire, dans le cadre de la rencontre annuelle entre Hosni Moubarak et les intellectuels égyptiens les plus en vue. A cette occasion, il critique publiquement le Président en lui attribuant la responsabilité du sousdéveloppement de l'Egypte, et réclame la préparation d'un nouveau texte constitutionnel, « (...) car la constitution de 1971 est arbitraire, et pour cette raison nous voulons une constitution complètement nouvelle dont les dispositions soient incontournables, qui garantisse l'équilibre des pouvoirs, assure l'indépendance judiciaire et réduise les pouvoirs du Président de la République. Parce que la situation actuelle vous donne des pouvoirs absolus et éternels, et cela est effrayant pour toute nation (...) ${ }^{16}$. Chercheur de haut niveau, reconnu pour ses vastes connaissances ainsi que « son aptitude à créer les formules et les

14. Il n'est pas inutile de rappeler au passage que - au moins jusqu'en 2011 - I'action politique des partis de l'opposition égyptienne se concentre essentiellement autour de leurs publications, cela à cause de l'Etat d'urgence en vigueur dans le pays depuis 1981 qui interdit toute activité en dehors des sièges des partis (meetings de rue etc.).

15. Voir : http://elbosla.org/

16. "Un intellectuel en face du Président », Al-'Arabî Al-Nâsirî, 18 octobre 2009. 
concepts innovateurs permettant de décrire, interpréter et synthétiser la réalité objective de nos vies et les grands changements qui caractérisent la région et le monde globalisé ${ }^{17}$, Mohammed Al-Sayyed Saïd est en même temps un intellectuel engagé, profondément concerné par l'état de la société qui l'entoure. Jadis militant marxiste, il est par la suite devenu un des plus vaillants défenseurs et promoteurs des droits de I'homme et a contribué à l'établissement des premières organisations qui œuvrent dans ce domaine ${ }^{18}$. Il est de fait considéré comme le "pionnier des droits de I'homme» non seulement en Egypte, mais aussi à l'échelle arabe ${ }^{19}$.

Soutenu par Mohammed AI-Sayyed Saïd et les militants du CESD, le projet d'Al-Badîl prend progressivement corps par le biais de plusieurs rencontres informelles auxquelles sont conviées d'autres personnalités de la gauche égyptienne. La présence d'acteurs relativement bien dotés en capital économique permet $d^{\prime}$ envisager la fondation $d^{\prime} u n$ journal quotidien, tandis qu'à l'origine l'idée était plutôt de lancer un hebdomadaire afin de limiter les coûts ${ }^{20}$. Mohammed Al-Sayyed Saïd défend le choix du support quotidien, estimant qu'il aura " un impact plus fort sur la vie politique et l'espace public $»^{21}$. Ces discussions préliminaires aboutissent, en mai 2006, à la création de l'entreprise de presse destinée à publier le journal, la "Société du progrès pour le journalisme, I'information et la publicité » (Charika al-Taqaddum li-lsahâfa wa-I-'i'lâm wa-I-'illân). Dotée d'un capital initial de 4 millions de livres égyptiennes, elle englobe 67 actionnaires, mais l'effort financier est essentiellement porté par trois individus qui représentent à eux-seuls $89 \%$ du total des fonds investis : Adel Al-Mashadd, Sabry Fawzy et Al-Sayyed Karaweya. Diplômés de I'Ecole Polytechnique de I'Université d'Alexandrie et du Caire, ces derniers sont des petits

17. Abdelfattah, 2009, p. 5.

18. Militant au sein de I'Organisation égyptienne des droits de I'homme (OEDH) depuis la fin des années 1980, il fait par la suite partie des fondateurs du Cairo Institute for Human Rights Studies : http://www.cihrs.org/?lang=en

19. Hasan, 2009, p. 3.

20. D'après la loi n'. 96 de 1996 sur l'organisation de la presse, le montant minimum du capital nécessaire à créer toute entreprise de presse doit s'élever à 1 million de livres égyptiennes pour un quotidien, 250.000 livres pour un hebdomadaire et 100.000 livres pour un mensuel. Par ailleurs, les frais $d^{\prime}$ impression et de distribution d'un hebdomadaire sont bien moindres que ceux engendrés par la publication d'un quotidien.

21. Entretien personnel avec FZ, Le Caire, août 2010. 
hommes d'affaires ${ }^{22}$ du secteur privé qui travaillent dans différents domaines d'activité. Or, ce portrait rapide des principaux financeurs du quotidien n'est pas exhaustif et se doit d'être complété par quelques informations supplémentaires concernant leurs trajectoires militantes.

Il est intéressant de noter que ces acteurs appartiennent, comme Mohammed Al-Sayyed Saïd et nombre d'autres fondateurs d'Al-Badîl, à la fameuse "Génération des années 1970 » (Iil al-sab înât) ${ }^{23}$. Nés durant la décennie 1950, socialisés par les structures jeunesse du régime nassérien, ils font partie de la cohorte très homogène qui a été éveillée à la politique suite à la défaite de juin 1967 contre l'armée israélienne. Comme maints autres gens du même âge, ils participent activement aux contestations estudiantines qui secouent le pays entre la fin des années 1960 et la décennie suivante ${ }^{24}$. Qui plus est, ils appartiennent à la même "unité générationnelle ${ }^{25}$ d'orientation marxiste, car parallèlement à l'activisme en milieu étudiant ils s'enrôlent dans différentes organisations communistes clandestines ${ }^{26}$. L'engagement dans des groupes concrets contribue de manière puissante à leur socialisation politique et à la formation d'une « conscience collective ${ }^{27}$ qui demeure un facteur rassemblant malgré la diversité des trajectoires ultérieures. Si nous n'avons pas la possibilité de nous attarder ici sur les itinéraires de ces acteurs après la période universitaire, retenons

22. Si nous ne disposons pas d'informations détaillées concernant les montants des capitaux de leurs respectives entreprises, nous pouvons vraisemblablement affirmer que ces trois personnalités ne font pas partie de la poignée d'entrepreneurs désignés par certains spécialistes de l'Egypte comme étant les "plus grosses fortunes du pays ». (Voir Kienle, 2002). Ils se définissent eux-mêmes en termes $d^{\prime}$ "«hommes d'affaires de condition moyenne», alors que d'autres protagonistes de l'expérience les qualifient de « petits hommes d'affaires d'orientation gauchiste » (entretien personnel avec SS, Le Caire, 27 juillet 2010). Par ailleurs, ils ne font pas partie des groupes de pression qui depuis les années 1980 représentent les intérêts des élites économiques du pays, tels que l'Egyptian Businessmen's Association (http://www.eba.org.eg) ou I'American Chamber of Commerce in Egypt (http://www.amcham.org.eg). 23. Pour une étude de l'émergence de cette génération politique et de sa trajectoire dans les années 1990-2000 voir : Al-Tohamy, 2009.

24. Sur le mouvement étudiant égyptien des années 1968-1973 voir: Abdallah, 1985, pp. 100-250.

25. Sur la notion d'unité générationnelle voir : Mannheim, 1990.

26. Les fondateurs d'Al-Badîl sont pour la plupart d'anciens membres du "Parti Communiste Egyptien des Travailleurs » (hizb al-'ummâl al-chuyûî̀ almisrî). Seule une petite minorité d'entre eux étaient affiliés au « Parti communiste du 8 janvier » (Al-hizb al-shuyû̂î - 8 yanayr) ou au Front trotskiste ('isba el-trûtskiyyn). Pour plus de détails sur ces formations marxistes clandestines, voir Gervasio, 2010, pp. 337-363.

27. Sur la notion de conscience collective, voir Mannheim, 1990. 
qu'ils ont été coupés pendant longtemps de la vie partisane. Comme la plupart des membres de leur génération, ils n'ont pas trouvé leur place au sein du Parti Tagammu', la seule formation de gauche à exister sur la scène politique légale du pays ${ }^{28}$. Certains $d^{\prime}$ entre eux ont reconverti le militantisme partisan des années de jeunesse vers d'autres sphères, telles que la mouvance des droits de l'homme ${ }^{29}$, d'autres se sont focalisés sur la mise en place de projets professionnels ou de vie. Ce n'est qu'à partir de la fin des années 1990 qu'ils reviennent progressivement au " politique » par le biais de certaines initiatives collectives, tout d'abord la "Célébration de la génération '70 » et les « Dialogues de I'avenir »30 et puis les mobilisations de la décennie suivante, telles que le Comité de coordination populaire de soutien à I'Intifada palestinienne, Kifâya et la Campagne populaire pour le changement ${ }^{31}$.

Au-delà des différences de parcours, ils demeurent reliés par un socle idéologique partagé, ce qui est d'autant plus intéressant lorsque I'on observe les "grands » actionnaires d'Al-Badîl. Ayant suivi des cursus d'ingénieurs, ces derniers ont par la suite démarré des activités entrepreneuriales en profitant des politiques de libéralisation économique initiées par Sadate et poursuivies par Moubarak. Bien qu'ils s'auto-définissent comme des hommes d'affaires de " condition moyenne », il est indéniable qu'ils appartiennent à une couche sociale

28. Certains d'entre eux ont essayé d'intégrer le Parti Tagammu' au début des années 1980, mais ils n'ont pas réussi à s'accommoder de la " gestion autoritaire » mise en œuvre par la direction centrale du parti et en sont sortis précocement.

A ce propos, voir Zahran, 2003. L'étude d'Ahmed Al-Tohamy (2009) sur la génération '70 montre bien la manière dont cette génération militante a été progressivement mise à l'écart de la scène politique partisane et comment, depuis le milieu des années 1990, ses membres ont commencé à chercher des voies pour revenir à s'impliquer en politique.

29. El-Khawaga, 1997.

30. La «Célébration de la génération '70 » (ihtifâl jîl al-sab'înât) est une manifestation organisée en février 1997 par d'anciens cadres marxistes du mouvement étudiant pour commémorer la révolte étudiante de 1972 et les émeutes du pain de 1977 et dont l'objectif était celui de se confronter et d'étudier la possibilité de lancer un nouveau projet politique de gauche. Sous le nom de "Dialogues de l'avenir », on désigne un travail de discussion et de réflexion politique qui, dans la deuxième moitié des années 1990, a impliqué des personnalités de la génération '70 appartenant à différents courants idéologiques (marxistes, nassériens et islamistes), et dont l'objectif était de trouver une entente commune à propos de la question nationale et de la crise politique égyptienne. Sur ces deux initiatives, voir Al-Tohamy, 2009, pp. $128 ; 217-220$.

31. Pour une présentation de ces mouvements par l'un des activistes engagés (et qui fait par ailleurs partie des fondateurs d'Al-Badîl) voir : Zahran, 2007. 
aisée, ou plus précisément à la classe de nouveaux riches ${ }^{32}$ émergée avec l'infitah. Cependant, leurs opinions politiques restent très proches de celles maturées pendant la période du mouvement étudiant, dans la mesure où ils se considèrent comme des intellectuels et militants de gauche et se montrent très critiques vis-à-vis du programme de privatisations mis en œuvre par le gouvernement égyptien pendant les années 2000.

Notons que cette double-identité, à la fois d'hommes d'affaires/ investisseurs et de gauchistes, ne sera pas sans effet sur l'expérience d'Al-Badîl, caractérisé d'emblée par une nature hybride. Ni journal partisan, ni journal privé, mais un peu les deux à la fois, le quotidien se distingue clairement de ses concurrents sur le marché de la presse. $\mathrm{S}^{\prime} \mathrm{il}$ est fondé et géré par une entreprise privée, ses propriétaires n'ont pas investi de l'argent pour obtenir des gains financiers, mais pour promouvoir des idées politiques qu'ils partagent. En même temps, Al-Badîl n'est pas non plus l'organe d'un parti au vrai sens du mot. $C^{\prime}$ est le produit d'un ensemble d'acteurs unis par un passé commun d'expériences politiques et par des liens d'amitié préexistants qui décident d'entamer une nouvelle aventure collective.

Encore faut-il examiner les motivations qui ont poussé ces acteurs à participer au projet. Les récits rétrospectifs des fondateurs témoignent à cet égard d'une volonté partagée de revenir à jouer un rôle plus concret dans la vie publique du pays, ou, comme le soulignent certains d'entre eux, de "s'ériger en porte-parole du peuple, de la majorité absolue des gens ${ }^{33}$ face aux dérives autoritaires du régime et aux politiques néolibérales menées par le gouvernement Nazif ${ }^{34}$. Tous mettent en avant l'importance de la conjoncture sociopolitique dans laquelle l'idée a pris naissance, à savoir le courant 20052006. Suite à l'émergence des mouvements "pro-démocratiques » de 2004, auxquels ces acteurs ont largement participé, la question du rôle et de la place de la gauche au sein du paysage politique égyptien semble se poser de manière plus urgente. II devient notamment impératif de remédier à la carence de visibilité dont elle souffre dans l'espace public national à un moment où ce dernier est en train de se reconfigurer sous l'effet de plusieurs facteurs, dont la prolifération des journaux privés et l'explosion des NTIC. Autrefois

32. J'emploie ici l'expression utilisée par Eberhard Kienle pour désigner les hommes d'affaires égyptiens ayant émergé avec les réformes d'ouverture économique. Voir Kienle, 2002.

33. Entretien personnel avec SF, Le Caire, août 2010.

34. Ahmed Nazif est nommé premier ministre en juillet 2004 à la tête d'un gouvernement technocratique dont la mission principale est celle d'accélérer les réformes libérales et mettre en œuvre un vaste programme de privatisations. 
tribune de la gauche dans son ensemble, voire pendant une période de I'opposition égyptienne au sens large du terme ${ }^{35}$, I'hebdomadaire Al-Ahâlî a abandonné depuis longtemps son attitude intransigeante vis-à-vis du pouvoir. Porte-parole officiel du Tagammu', ou plus précisément de sa direction centrale, il reflète le positionnement d'un parti taxé de connivence avec le régime de Moubarak par de nombreux gauchistes, $y$-compris les fondateurs d'Al-Badîl qui $\mathrm{n}^{\prime}$ hésitent pas à le qualifier $\mathrm{d}^{\prime}$ « instrument de confiscation de la vie politique ${ }^{36}$. Cela dit, le nouveau journal n'est pas seulement conçu en tant qu'outil médiatique devant pallier à un prétendu manque de visibilité, mais il est pensé comme un instrument de réalisation d'un objectif beaucoup plus ambitieux : " construire un mouvement dans la société ", "forger la troisième voie alternative à la fois au Parti national (démocratique) et aux Frères musulmans », ou encore " créer la troisième voie civile ${ }^{37}$. Ces énoncés étant lourds de sens, il s'agit tout d'abord de revenir sur leur contexte de production afin de mieux en apprécier la portée historique. Recueillies lors d'entretiens menés en 2010, c'est-à-dire avant le soulèvement de 2011 et l'entrée des Frères musulmans sur la scène politique légale, ces affirmations sont profondément révélatrices des craintes ressenties par une partie de la gauche égyptienne quant à la montée en puissance du courant islamiste sur la scène publique du pays, notamment après les élections législatives de l'automne $2005^{38}$. De surcroît, elles montrent que les espoirs de consensus et d'unité de l'opposition matérialisés un an plus tôt par Kifâya se sont rapidement éteints.

\section{AL-BADÎL «ÉCHAPPE » AUX FONDATEURS ... CHRONIQUE DU PASSAGE D'UNE GÉNÉRATION À L'AUTRE}

Al-Badîl paraît pour la première fois en juillet 2007 après une longue phase préparatoire marquée entre autres par d'énormes difficultés à obtenir sa licence de publication. Le retard avec lequel I'autorisation est délivrée révèle que les autorités ne sont pas forcément

35. A la fin des années 1970, I'organe du Parti Tagammu' ouvra ses pages aux intellectuels d'autres courants idéologiques qui ne disposaient pas de canaux d'expression propres, tels que les militants du Parti Néo-Wafd.

36. Entretien personnel avec I'un des fondateurs, Le Caire, août 2010.

37. Entretiens avec plusieurs fondateurs, Le Caire, août 2010 ; avril 2011.

38. Lors des élections de 2005, les Frères Musulmans parviennent à remporter 88 sièges, ce qui représente un résultat historique si l'on considère les pratiques d'interférence mises en place par le régime lors des compétitions électorales. 
à l'aise face à l'émergence de cette voix "alternative $»^{39}$. Quotidien national d'information publié en seize pages, Al-Badîl se veut une publication sérieuse et se place d'emblée au " pôle intellectuel » du champ journalistique égyptien, à savoir du côté opposé par rapport aux journaux à sensation qui fleurissent dans le pays depuis la fin des années $1990^{40}$. En effet, il ne donne pas beaucoup d'importance aux faits divers et néglige les nouvelles sur la vie des célébrités égyptiennes qui font souvent la Une dans les tabloïds ${ }^{41}$. Ses domaines d'information privilégiés sont I'actualité politique domestique et internationale, la société, la culture, l'art et l'économie, sans oublier le sport puisqu'il est susceptible $d^{\prime}$ intéresser son lectorat cible ${ }^{42}$. Par ailleurs, il se distingue également par la présence d'une rubrique quotidienne consacrée au journalisme d'investigation, témoignant d'une volonté claire d'exercer la fonction de "chien de garde " traditionnellement attribuée à la presse dans le monde anglo-saxon.

Une analyse du contenu du journal au fil de sa parution montre que les sujets les plus traités portent sur des questions telles que la pauvreté et la hausse des prix, la torture et les abus policiers du régime, les affaires de corruption où sont impliqués aussi bien de hauts responsables de l'Etat que des membres des élites économiques, la malgouvernance et le dysfonctionnement des services publics. D'autre part, le quotidien accorde une très large couverture aux mouvements sociaux et contestataires qui bouleversent l'Egypte en 2007 et 2008, s'intéressant aussi bien aux collectifs focalisés sur des questions de

39. La licence de publication est obtenue en avril 2007 alors que la demande avait été déposée auprès du Haut Conseil de la Presse (HCP) en août 2006. Or, d'après la loi nº 96 de 1996 sur l'organisation de la presse le HCP dispose d'un délai de deux mois pour examiner les dossiers reçus, suite auquel il est tenu de donner une réponse. Au début de 2007, après une attente de plusieurs mois pendant lesquels ils ne parviennent à avoir aucune information concernant leur licence, les fondateurs d'Al-Badîl décident d'intenter un procès contre le HCP avec le soutien de l'Arabic Network for Human Rights Information, ONG égyptienne qui travaille dans le domaine de la liberté d'expression et de publication. Ils préparent la documentation nécessaire, mais avant que le procès ne commence ils obtiennent I'autorisation à publier. Entretien avec le directeur exécutif de l'ANHRI, Le Caire, mai 2011.

40. Sur le phénomène de la «presse jaune » en Egypte voir : Amir, 2006, pp. 115-128.

41. Le manifeste éditorial d'Al-Badîl insiste à plusieurs reprises sur la nécessité que le futur journal s'éloigne du sensationnalisme, de la vulgarité et du ton provocateur qui caractérisent la grande majorité des publications privées égyptiennes ainsi que sur l'importance de savoir convaincre le lecteur à travers l'emploi d'arguments rationnels.

42. Le manifeste des fondateurs précise à ce propos qu'Al-Badîl, loin de se contenter de l'élite intellectuelle du pays, vise un large public. 


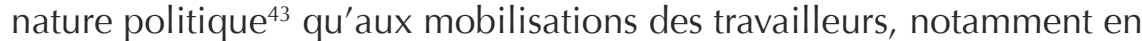
milieu ouvrier ${ }^{44}$, mais aussi aux groupes écologistes.

Dès sa parution sur le marché, Al-Badîl s'affirme comme l'une des voix les plus critiques vis-à-vis des autorités en place. Journal à l'orientation politique précise, il offre des tribunes hebdomadaires ou quotidiennes à plusieurs intellectuels et militants de la gauche égyptienne. En même temps, il confirme son " appartenance à la société civile égyptienne et mondiale $»^{45}$ en lançant des campagnes de promotion des droits de l'homme, en publiant des rapports d'ONGs qui travaillent dans ce domaine et en proposant un espace d'expression à leurs activistes. En plus du fait qu'ils écrivent des articles d'opinion ou des dossiers portant sur des sujets sensibles tels que la liberté d'expression ou la torture, ces derniers sont très souvent cités comme sources par les journalistes d'Al-Badîl. Qui plus est, les éditoriaux que Mohammed Al-Sayyed Saïd publie tous les jours en dernière page se focalisent fréquemment sur la question des droits de I'homme et l'absence ou le non-respect dont ils font l'objet en Egypte, avec un accent particulier sur les griefs et les attentes des groupes les plus défavorisés et marginalisés de la population.

Observé dans une perspective diachronique, le journal papier ne montre pas de mutations majeures dans sa structuration interne ou dans les thèmes traités. Les rubriques quotidiennes retiennent leur place tout au long de l'expérience et seules celles hebdomadaires tendent à disparaître après quelques mois, signe qu'une hyperspécialisation journalistique $n^{\prime}$ est pas viable dans un quotidien aux ressources limitées tel qu'Al-Badîl ${ }^{46}$. II en va de même pour les sujets privilégiés

43. Outre Kifâya et les autres mouvements "pour le changement " (Journalistes, avocats, etc.), I'on mentionnera le "Mouvement du 9 mars pour l'indépendance des universités » et les collectifs «Egyptiens contre la torture », "Egyptiens contre la discrimination religieuse », " Ingénieurs contre la tutelle » et "Médecins sans droits ".

44. Pendant les premiers mois de son expérience, Al-Badîl publie même une rubrique hebdomadaire intitulée 'Ummâl. Bien qu'elle disparaisse par la suite, le journal continue d'accorder aux mobilisations ouvrières une large place à l'intérieur de ses pages. Par ailleurs, Al-Badîl est le premier quotidien en Egypte à avoir mis en place, au sein du service des « infos géné », une cellule de reporters spécialisés sur les questions ouvrières, faisant de celles-ci une véritable priorité éditoriale.

45. Comme le déclare le manifeste éditorial du journal.

46. Comparé à d'autres quotidiens privés qui paraissent en Egypte dans les années 2000, Al-Badîl se caractérise par un capital initial assez réduit. Quatre millions de livres égyptiennes sont un montant ridicule si on les compare aux 16 millions LE investis par les fondateurs d'Al-Masrî Al-Yaum en 2004, ou encore aux 120 millions LE investis en 2008 par les fondateurs d'Al-Shurûq. 
du journal, qui font l'objet de transformations mineures, tandis que les quelques évolutions remarquables concernent la mise en page et la manière de présenter l'information. La maquette du quotidien se transforme progressivement pour accroître son attractivité, la Une devient au fur et à mesure plus lisible et organisée, et les titrailles se font plus accrocheuses à travers l'emploi généralisé d'expressions dialectales. Autrement dit, le quotidien change de personnalité et prend une allure plus dynamique et captivante, même si, ce faisant, il perd en sobriété. Or ces transformations stylistiques, loin d'être négligeables, sont concomitantes avec la montée en puissance d'une "nouvelle génération" au sein de la rédaction d'Al-Badîl. Décelables entre autres par une observation fine de l'ours ${ }^{47}$ du journal, les changements successifs que subit l'organigramme sont révélateurs du rajeunissement progressif de son comité de rédaction. Cette question mérite que l'on s'y attarde quelque peu. Dans les paragraphes qui suivent, j'essaierai de rendre compte de ce processus de passage d'une génération à l'autre et de mettre en lumière les différents enjeux qui y sont liés. Mais commençons d'abord par présenter les différents acteurs qui participent à l'expérience journalistique.

Lors de sa parution sur le marché, Al-Badîl est géré par un ensemble d'individus appartenant à ladite génération '70. Déjà en " position de force » au moment de la fondation, en raison de leur nombre et du rôle central qu'ils jouent financièrement et intellectuellement, ces acteurs traduisent spontanément leur influence en pouvoir décisionnel et managérial. Sans qu'ils n'aient nécessairement d'expérience préalable significative dans le domaine de la presse, ils vont occuper la plupart des fonctions importantes au sein de l'administration et de la rédaction du journal. Il en est ainsi par exemple des membres du CDA d'A/Badîl, qui font tous partie du groupe susmentionnét8. L'autorité dont ils jouissent en matière de gestion technique et financière est bien sûr directement proportionnelle aux montants qu'ils ont investis dans la société Al-Taqaddum et à leurs compétences d'entrepreneurs, mais ils n'en demeurent pas moins des novices dans l'industrie médiatique,

47. En jargon journalistique, I'ours désigne l'endroit où, dans une publication, sont répertoriés les noms et fonctions des collaborateurs (rédaction, services commerciaux et administratifs) et celui de l'imprimeur.

48. Il s'agit des trois majeurs actionnaires du journal, dont nous avons déjà parlé plus haut, et d'un quatrième associé minoritaire. A noter que le CDA sera ensuite élargi à la fin de 2008 pour englober trois autres membres, deux desquels appartiennent aussi à la génération '70. 
leurs carrières s'étant pour l'essentiel déroulées dans d'autres secteurs ${ }^{49}$. De surcroît, la " génération '70 " tient une place significative au sein de la rédaction du journal, où elle occupe presque tous les postes managériaux. Mis en place par Mohammed Al-Sayyed Saïd, le premier comité de rédaction est très parlant à cet égard, dans la mesure où il englobe plusieurs activistes et écrivains du même âge qui proviennent des files de la gauche. Anciens compagnons de route, ces acteurs se connaissent depuis longtemps et au fil des ans ont partagé plusieurs aventures intellectuelles et militantes.

A côté de cet ensemble somme toute restreint de gens ayant dépassé la cinquantaine, l'équipe rédactionnelle d'Al-Badîl se caractérise par la présence d'une "armée ${ }^{50}$ de reporters âgés de vingt à trente-cinq ans. Le choix de recruter des éléments jeunes et inexperts semble d'abord être lié à l'ambition des fondateurs de "former une nouvelle génération de journalistes aux méthodes, à l'éthique et aux genres de la presse démocratique moderne (...) $»^{51}$. En effet, comme l'affirme la responsable du service "Société » du quotidien, « former des jeunes est relativement simple, car leur esprit est ouvert et n'est pas conditionné par les mauvaises habitudes développées durant des années d'exercice du métier ${ }^{52}$. II reste qu'Al-Badîl est une publication "pauvre » et n'a pas les moyens d'embaucher un large nombre de professionnels expérimentés.

Notons que la "jeunesse d'Al-Badîl » est un motif très récurrent dans les récits des acteurs interviewés. Les fondateurs du quotidien soulignent à plusieurs reprises que « $C^{\prime}$ était une expérience jeune, voire très jeune », qu'Al-Badîl était "plein de jeunes passionnés » et que leur présence était importante, car « nous-mêmes (les fondateurs) nous avions besoin de leur enthousiasme et de leur dynamisme $»^{53}$. Quant aux enquêtés les moins âgés, ils emploient eux-aussi ce leitmotiv et mettent en avant l'idée d'un lien causal direct entre le poids numérique des jeunes en salle de rédaction et le climat détendu et amical qu'on y

49. L'un d'entre-deux a mis en place une usine de matériel électrique, tandis que deux autres travaillent dans le secteur de la construction et du bâtiment. Le quatrième membre, associé minoritaire, est propriétaire d'une petite maison d'édition.

50. Pour citer le mot employé par l'un des fondateurs du journal, qui affirme : "Nous avions une armée de (journalistes) très jeunes » (jaych min al-chabâb al-saghîr). Entretien personnel, avril 2011.

51. Manifeste éditorial d'Al-Badîl.

52. Entretien personnel avec KK, Le Caire, août 2010.

53. Entretien personnel avec SK, avril 2011. 
respirait ${ }^{54}$. Au-delà de ces portraits d'une jeunesse déclinée au singulier, il s'agit de rappeler, à la suite de Pierre Bourdieu, que les journalistes d'Al-Badîl ne constituent pas a priori un groupe social homogène doté $d^{\prime}$ un intérêt commun ${ }^{55}$. Au contraire, ils se différencient entre eux selon plusieurs critères, dont le milieu d'origine, la provenance géographique, le parcours scolaire et universitaire, mais aussi l'âge, l'expérience professionnelle préalable et le positionnement hiérarchique au sein de la rédaction. Parmi ces "jeunes", certains viennent tout juste de terminer leurs études, ou bien ils sont encore à l'université, et sont donc recrutés au journal comme stagiaires. D'autres, un peu plus âgés, ont déjà quelques expériences dans le domaine, ce qui leur permet d'être employés en tant que rédacteurs ou correspondants à tous les effets. Enfin, une poignée d'entre eux ont commencé leur carrière à la fin des années 1990 et disposent des compétences nécessaires pour accéder à des postes relativement importants.

Malgré les différences de trajectoires sociales et de positionnements dans l'organigramme du journal, des solidarités s'établissent rapidement entre eux sur la base de deux facteurs principaux : d'une part, ils ont un âge où les amitiés se nouent facilement; d'autre part, ils sont unis par une attitude politique commune. Le groupe se caractérise en effet par un haut degré d'intérêt pour les affaires publiques et la présence en son sein d'un fort noyau de militants opposés au régime, sans qu'ils n'aient pour autant la même orientation idéologique. Si la plupart d'entre eux sont de gauche, d'autres se définissent comme étant des " libéraux » et parmi les plus jeunes certains se disent islamistes. Les parcours militants de ces acteurs sont extrêmement diversifiés et il n'est pas facile d'en faire une catégorisation précise. Toutefois, quelques caractéristiques communes se dégagent aisément. Disons tout d'abord que la plupart d'entre eux n'ont pas été socialisés au sein de partis politiques et ne revendiquent aucune affiliation partisane ${ }^{56}$, mais que plusieurs ont vécu des expériences de collaboration plus ou moins longues et importantes avec des organisations des droits de l'homme et se considèrent comme étant des activistes de la "société civile ". D'autre part, il faut également souligner qu'un grand nombre de ces reporters ont participé à l'expérience de Kifâya et des Jeunes pour le changement ${ }^{57}$. Ils sont devenus des habitués des manifestations,

54. Les phrases «Nous étions tous jeunes et n'étions pas que des collègues, nous étions des amis " reviennent constamment dans les entretiens réalisés avec ces journalistes, notamment lorsqu'ils décrivent I'ambiance de travail au sein de la rédaction.

55. Bourdieu, 1984.

56. D'après plusieurs enquêtés, seuls neuf journalistes sur un total de quatre-vingt-seize étaient affiliés à des structures partisanes de gauche.

57. Sur l'expérience des Jeunes pour le changement voir Hassabo, 2009. 
des marches et des sit-in dans l'espace public, ce qui les a amenés à expérimenter de première main les pratiques répressives des forces de sécurité égyptiennes. Liés par un commun sentiment d'opposition visà-vis du régime autoritaire de Moubarak, ils appartiennent à ce qu'on pourrait définir en termes de "génération politique ${ }^{58}$ en gestation", qui ne se révélera à soi-même et au monde qu'en 2011.

Rattachés aux différents services de la rédaction, les " jeunes» reporters s'approprient en quelque sorte le journal, l'imprègnent de leur énergie et vivacité et en font un espace de sociabilité peu conforme à l'image qu'on a d'un simple lieu de travail. Le siège du journal devient pour eux une deuxième maison. Ils y passent tout leur temps et ne s'en éloignent que le soir pour rentrer chez eux. La phrase "On arrivait au journal à dix heures du matin et on repartait à dix heures du soir, car on aimait le lieu » revient à plusieurs reprises dans les récits des enquêtés. Mais cette appropriation symbolique du siège du journal s'accompagne aussi de la " conquête » des postes les plus élevés dans la hiérarchie de la rédaction. La montée en puissance des « jeunes » au sein d'Al-Badîl est un processus graduel qui s'achève symboliquement en octobre 2008 lorsque Khaled Al-Balshy, alors âgé de 36 ans, devient rédacteur-en-chef du quotidien suite à la démission de Mohammed AlSayyed Saïd pour des raisons de santé. A ce moment-là, le comité de rédaction du journal est entre les mains d'une nouvelle génération, car tous ses membres sont nés dans les années $1970^{59}$. Comment s'opère ce passage d'une génération à l'autre et qu'est-ce que cela implique ? S'agit-il d'un processus conflictuel, étant donné que la passation des fonctions entraîne aussi un transfert de pouvoir décisionnel et managérial?

S'il n'y a pas, à proprement parler, de conflit ouvert entre les deux groupes d'âge, remarquons que le "fossé » intergénérationnel est large, dans la mesure où leurs contextes sociohistoriques de formation sont très différents. Les membres de la " génération '70 » ont grandi sous le régime nassérien et leur socialisation s'est faite dans la période des politiques socialistes par le biais des structures d'encadrement dont I'Organisation de la jeunesse socialiste, qui était fondée sur un modèle de type soviétique. Ainsi, ils sont en quelque sorte un "produit » de la révolution de 1952 et du régime militaire des Officiers libres,

58. Au sens où I'entendent Richard et Margaret Braungart : « il y a génération politique lorsqu'un groupe d'âge historique se mobilise pour œuvrer au changement social ou politique ». Braungart R. \& M., 1989, p. 9.

59. Le comité de rédaction du journal comprend, outre le rédacteur-enchef, le directeur de rédaction (mudîr al-tahrîr), deux secrétaires de rédaction et le directeur artistique. II faut aussi souligner que la plupart des responsables de services appartiennent à la même cohorte. 
bien qu'après la défaite de 1967 ils aient participé aux contestations estudiantines contre le régime.

Au contraire, les "jeunes » d'Al-Badîl ont grandi dans une période de libéralisation économique et de remise en cause du nassérisme, ils ont " toujours ou presque toujours vécu sous l'état d'urgence en vigueur depuis l'assassinat de Sadate en 1981 et n'ont connu - ou presque - qu'un seul président, Hosni Moubarak ${ }^{60}$. Ils n'ont pas été encadrés par des structures corporatistes et sont rentrés à l'université dans une période de délibéralisation politique où les campus étaient strictement contrôlés par les forces de sécurité et l'activisme en milieu étudiant était très faible ${ }^{61}$. Et pourtant, il est nécessaire de rappeler que ces acteurs ont aussi grandi à l'époque de la révolution de l'information et de la communication, de l'explosion des chaînes satellitaires et de I'internet, dans une période de "libération de la parole », si l'on peut ainsi dire.

Les deux groupes d'âge sont donc tributaires de conditions sociales, politiques, économiques et culturelles très différentes. Cela se reflète d'abord dans des façons assez diverses de construire leur rapport au politique. Les " jeunes» ne sont en général pas des militants au sens traditionnel du terme, ils n'ont pas été socialisés au sein de formations partisanes et sont d'une certaine manière éloignés du 'dogmatisme' qui caractérise leurs confrères plus âgés. Mais il faut également dire que le fossé intergénérationnel se traduit dans deux manières différentes de concevoir l'identité de journaliste. Plus des intellectuels de gauche que des journalistes au sens strict du terme, les membres de la génération '70 sont là en vertu de leur appartenance à un même courant politique et considèrent le quotidien comme un support de promotion de leur projet idéologique. Aussi peut-on considérer qu'ils restent liés à une conception "traditionnelle» de l'intellectuel-journaliste dont la mission essentielle est celle d'éduquer les masses. Le manifeste éditorial d'Al-Badîl est révélateur de cela, dans la mesure où il insiste à plusieurs reprises sur le rôle éclairant (tanwîrî) et éducatif (tathqîîi) du futur quotidien et sur la nécessité de donner au " peuple " l'accès à une culture créative qui puisse le transformer en acteur du changement historique. L'on remarquera que le choix du terme tathqifî́ n'est pas anodin. Il évoque le maître mot des politiques culturelles nassériennes des années $1960^{62}$, qui ont sans doute eu un fort impact sur l'imaginaire de ces gens alors adolescents. Ces acteurs sont porteurs d'une conception

60. Hassabo, 2009.

61. Voir Shehata, 2011.

62. Sur les politiques culturelles des années 1960 voir Gonzalez-Quijano, 1998. 
du journalisme où le " message » (risâla) idéologique prime sur toute autre considération ${ }^{63}$. Au contraire, les jeunes reporters d'Al-Badîl s'identifient en termes de professionnels de l'information et paraissent s'intéresser plus à la recherche de l'information exclusive et à l'emploi de procédés stylistiques et linguistiques visant à frapper l'esprit du lecteur qu'à la nécessité de porter un message éducatif et éclairant. Pour le dire en d'autres mots, ils sont plus sensibles aux mécanismes du marché et à ses règles concurrentielles. Cela ne peut s'expliquer qu'en prenant en considération les transformations du champ journalistique égyptien à partir des années 1990 et la réapparition d'un régime de concurrence au sein du marché de la presse, avec notamment l'émergence des médias privés. L'on pourrait avancer que les jeunes journalistes d'AlBadîl sont un " produit » de la reconfiguration du champ journalistique dans la mesure où leur socialisation professionnelle s'est faite dans cette période de mutations. Ils sont entrés dans la profession à une époque où il y a un choix très large d'institutions de presse où se former. Ils ont d'ailleurs souvent multiplié les expériences en passant d'une rédaction à l'autre, en travaillant au sein de deux ou trois publications à la fois et dans différents types de journaux, non seulement du point de vue de la périodicité (quotidienne, hebdomadaire ou mensuelle), mais aussi du point de vue de la propriété (journaux étatiques, partisans ou privés), sans oublier que certains d'entre eux ont aussi travaillé au sein des nouvelles chaînes satellitaires égyptiennes, telles que Dream ${ }^{64}$. Ces multiples expériences, en particulier dans les médias privés, leur ont donné des compétences diversifiées, les ont rendus plus attentifs aux modalités de fonctionnement du marché et les ont habitués à un journalisme libre des contraintes politiques imposées par les autorités au pouvoir, dans le cas des publications étatiques, ou par la direction du parti, dans le cas des journaux partisans.

La rédaction d'Al-Badîl devient ainsi rapidement un espace de luttes symboliques entre les deux générations préalablement identifiées. Les jeunes reporters rentrent dans des relations de concurrence avec leurs confrères plus âgés en mettant en question leur "légitimité » à la tête du journal. Vus à travers leurs yeux, les fondateurs du quotidien disposent certes d'un « capital symbolique »-au sens bourdieusien du terme - plus élevé, mais il n'en demeure pas moins que leur notoriété et reconnaissance sont plus liées au champs militant et intellectuel qu'au domaine journalistique. L'arrivée d'une nouvelle génération au sommet de la rédaction d'Al-Badîl va graduellement imprimer des transformations au quotidien. Si ces changements concernent moins les choix thématiques que ceux stylistiques, il reste que les

63. Entretien avec MZ, Le Caire, août 2010.

64. Sur la naissance de la chaîne Dream voir Hamdy, 2002. 
fondateurs du journal auront la sensation de perdre le contrôle sur le projet éditorial. Plusieurs d'entre eux affirment à ce propos que le journal serait progressivement devenu "populiste » (cha'abawi) et "sensationnaliste» (muthîr), ce qui l'aurait éloigné des objectifs fixés au moment de la fondation. Certains considèrent par ailleurs qu'il aurait progressivement abandonné la ligne éditoriale qui le caractérisait au début et qu'il aurait ainsi cessé de fonctionner en tant qu'instrument de promotion de la " troisième voie " alternative au PND et aux Frères musulmans.

\section{AL-BADÎL AL-JADÎD : UNE VOIX «RÉVOLUTIONNAIRE »?}

Après avoir analysé l'expérience d'Al-Badîl du point de vue des acteurs impliqués, il s'agit maintenant de focaliser le regard sur ce qu'on pourrait nommer la «deuxième vie » du journal. Contraint à la fermeture en avril 2009, officiellement pour des raisons financières ${ }^{65}$, ce dernier est relancé un an et demi après sous un nouveau nom et un nouveau format: le $1^{\text {er }}$ novembre 2010 naît Al-Badîl Al-Jadî́ ( $L a$ Nouvelle Alternative), site web d'information qui se présente comme étant la "réincarnation» de son prédécesseur. Comme I'affirme un article publié le jour de son lancement: «Lundi $1^{\text {er }}$ novembre, à sept heures du soir, le rêve d'Al-Badîl revient une nouvelle fois à la publication après une interruption d'environ un an et demi à partir du 4 avril 2009. Du Syndicat des journalistes, Al-Badîl revient sous format électronique avec sa politique éditoriale qui met la profession au dessus des « lignes rouges » et place le lecteur au dessus de toute autre considération. (...) Al-Badîl revient à ses lecteurs et à la communauté journalistique pour remplir son rôle à la cour de Sa Majesté66 et ouvre de nouveaux horizons au journalisme en tant que profession, art et devoir national indispensable $»^{67}$. Les discours prononcés par les personnalités intellectuelles invitées à la cérémonie d'inauguration du site, qui se tient dans les locaux du Syndicat des journalistes, insistent de façon explicite sur l'importance du retour d'Al-Badîl dans I'espace médiatique égyptien et sur le lien de continuité entre le nouveau site web et l'ancien journal ${ }^{68}$. Dans les jours qui suivent, des articles

65. D'après ses administrateurs, en moins de deux ans le journal aurait subi des pertes de l'ordre de seize millions de livres égyptiennes.

66. L'expression "sâhibat al-galâla » est parfois employée en Egypte pour désigner la presse.

67. "Parce que les rêves ne meurent pas: "Al-Badîl " revient à ses lecteurs ", $1^{\text {er }}$ novembre 2010 : http://www.masress.com/elbadil/588

68. Voir : "Des lecteurs, des journalistes et des politiciens célèbrent le naissance d'Al-Badîl : ton rêve est notre rêve », 3 novembre 2010 :

http://elbadil.com/2010/11/03/15229/ 
signés par des intellectuels et journalistes qui avaient été impliqués d'une manière ou d'une autre dans la vie du quotidien célèbrent la naissance de La Nouvelle Alternative en mettant I'accent sur le motif du «retour ». L'on citera à titre d'exemple Ahmad Baha' Al-Din Shaaban, qui affirme à ce propos : "Al-Badîl est revenu, non pas pour commencer son chemin du point zéro, mais pour reprendre un long voyage commencé sur le papier durant lequel il a laissé une empreinte claire, que nous n'oublions pas, sur la vie politique, culturelle et journalistique égyptienne ${ }^{69}$.

Or la centralité que prennent les thèmes du "retour » et de la " continuité » dans les discours entourant la parution d'Al-Badîl A/Jadîd occulte en réalité l'existence d'une fracture évidente entre les deux expériences, ce que révèle d'emblée le nom du site web. L'usage de l'adjectif «jadîd» n'est en ce sens pas anodin : soulignant qu'il s'agit de quelque chose de "neuf », c'est un marqueur de différence qui vise à creuser l'écart entre un ancien et un nouveau, un avant et un après.

Que I'on ne s'y trompe pas : il existe un lien assez fort entre les deux médias et la naissance d'Al-Badîl Al-Jadîd est profondément ancrée dans l'expérience de son prédécesseur, dans la mesure où les acteurs qui le créent se sont rencontrés au sein de la rédaction du quotidien et ont tissé entre eux des liens de solidarité durables qui fondent la cohésion de leur groupe et la possibilité même d'imaginer et mettre en œuvre un nouveau projet médiatique. En effet, le site web est conçu et lancé par une poignée de journalistes qui travaillaient à Al-Badîl et qui, suite à la fermeture de celui-ci, n'ont jamais perdu l'espoir de pouvoir le ramener à la vie. Mais La Nouvelle Alternative n'est plus L'alternative : aucun des fondateurs du quotidien ne joue un rôle dans la création du site web et même si certains d'entre eux y publient de temps en temps des articles d'opinion, ils ne sont aucunement impliqués dans la gestion du projet ni dans le travail rédactionnel quotidien. Par ailleurs, Al-Badîl Al-Jadîd est rapidement investi par un groupe assez large de néo-diplômés et étudiants qui ne sont pas passés par l'expérience du journal papier, si bien qu'après quelques mois l'équipe du site semble être presqu'entièrement renouvelée.

Le père spirituel et matériel d'Al-Badîl Al-Jadîd est Khaled Al-Balshy. $C^{\prime}$ est lui qui, en août 2010, prend I'initiative de ressusciter le journal à travers la création d'un site web d'information et pourvoit au financement du projet en utilisant l'argent obtenu de la vente d'un terrain de propriété.

69. "La bataille d'Al-Badîl", 4 novembre 2010 :

http://elbadil.com/2010/11/04/30546/ 
Né en 1972 à Menoufiya, diplômé de la Faculté de communication (département de journalisme) de l'Université du Caire, Khaled Al-Balshy entame sa carrièreen 1996 à Al-Yasâr, revue politique mensuelleéditée par le Parti Tagammu', où en quelques mois il devient secrétaire de rédaction. Par ailleurs, il intègre l'équipe rédactionnelle de l'hebdomadaire Rose Al-Yousef, où il travaillera, avec quelques interruptions, jusqu'à 2005. Pendant la même période, il dirige la rédaction de plusieurs revues à parution irrégulière éditées par des organisations de la société civile, telles que Kalâm Sinâ'iya ${ }^{70}$. La collaboration avec les ONGs se poursuit dans la nouvelle décennie et lui permet de s'intéresser de plus près aux questions des droits de l'homme et en particulier aux conditions de vie des ouvriers égyptiens. Dans les années 2000, de nouvelles expériences professionnelles l'amènent d'abord à travailler à la chaîne privée Dream en tant que préparateur d'émissions. Puis, en 2005, il fait partie de I'équipe de journalistes qui relancent I'hebdomadaire indépendant AlDustûr $r^{71}$ sous la direction d'Ibrahim Eissa, et devient rédacteur-en-chef adjoint de la publication. Il démissionne du journal pendant l'été 2006 à cause de divergences avec Eissa et avec le conseil d'administration, mais il est tout de suite recruté à Al-Badîl comme responsable du service des enquêtes, entre autres parce que durant son parcours professionnel il a développé un goût particulier pour le journalisme $d^{\prime}$ investigation $^{72}$. Son investissement total dans le travail au quotidien ${ }^{73} \mathrm{I}^{\prime}$ amène $\mathrm{d}^{\prime}$ 'abord, en mars 2008, à devenir directeur de rédaction (mudîr al-tahrîr) et puis, en octobre de la même année, rédacteur-en-chef du journal. Parallèlement à la profession de journaliste, il participe à la vague de contestation politique qui traverse l'Egypte depuis le début des années 2000. Membre de Kifâya et du groupe Journalistes pour le changement, il est considéré comme un opposant du régime et se revendique lui-même comme étant proche de la pensée de gauche sans pour autant militer au sein de structures partisanes.

70. Cette publication est éditée par le Centre de Prestations Syndicales et Ouvrières de Helwân (Dâr al Khadamât al Niqâbya wa al 'Ummâlya), ONG spécialisée dans les questions du travail et du syndicalisme ouvrier.

71. Al-Dustûr paraît pour la première fois en 1996 en tant que publication à licence chypriote. Dirigé par Ibrahim Eissa, alors âgé de trente et un ans, il se caractérise par une liberté de ton inédite dans l'espace journalistique du pays. II sera aussitôt fermé par les autorités égyptiennes. Sur la première expérience d'Al-Dustûr voir : El-Khawaga, 2000.

72. Entretien personnel, Le Caire, avril 2011. A noter qu'en 2005 le Syndicat des journalistes lui a conféré le prix annuel pour la meilleure enquête journalistique pour une série d'articles sur les risques cancérigènes de l'amiante et ses victimes.

73. Plusieurs acteurs ayant participé à I'expérience d'Al-Badîl le décrivent comme un " travailleur infatigable ", comme " celui qui arrive le premier au bureau et qui part toujours le dernier ». 
C'est lui qui, suite à la fermeture d'Al-Badîl, dirige les multiples initiatives visant à ressusciter le journal. II joue d'abord le rôle d'intermédiaire entre les administrateurs et les reporters lors des négociations autour de la possibilité de relancer Al-Badîl sous format hebdomadaire. Dans un deuxième temps, il part à la recherche de potentiels investisseurs prêts à injecter de l'argent dans la société AlTaqaddum ou bien à la racheter. Ces tentatives, qui s'étalent sur plus d'un an, n'auront pas de succès et il faudra attendre août 2010 pour que la décision de lancer le site-web soit enfin prise. C'est alors que le directeur exécutif de I'ANHRI ${ }^{74}$, Gamal Eid, contacte Khaled Al-Balshy et lui propose de créer une publication électronique avec le soutien de son organisation. Le journaliste accepte et se met immédiatement au travail en prenant contact avec ses anciens collègues afin de constituer une première équipe rédactionnelle. Encore faut-il trouver un mécène intéressé à pourvoir au financement du projet car, si les coûts techniques sont extrêmement faibles, les frais en matière de ressources humaines restent élevés. A cet effet, Al-Balshy prend contact avec I'un des hommes d'affaires qui avaient participé à la fondation d'Al-Badîl et l'invite à participer au nouveau projet, l'idée étant aussi de remettre en ligne l'archive électronique du journal qui avait disparu immédiatement après sa clôture. Bien que les premières négociations semblent avoir quelques succès, il ne parvient pas à l'impliquer. "Mais on avait déjà travaillé à la préparation du site ", dit-il,, "et j'étais convaincu qu'à ce moment-là il fallait être présents (dans l'espace médiatique). Donc j'ai décidé de poursuivre mon projet et je me suis dit : 'Si je ne peux pas faire L'Alternative, alors je vais faire La Nouvelle Alternative' $»^{75}$. La création du site web marque donc en quelque sorte la "rupture » définitive entre les journalistes et les fondateurs du quotidien et porte à son comble le processus d'appropriation d'Al-Badîl par ses jeunes reporters. Du reste, un article publié le jour du lancement du site proclame qu' «Al-Badîl revient grâce un rêve né de l'initiative des journalistes, ses véritables artisans »(c'est moi qui souligne), propos qui n'est à l'évidence pas dénué de portée polémique.

Sans avoir trouvé de financeurs, La Nouvelle Alternative s'appuie dans un premier moment sur le travail bénévole d'un certain nombre de journalistes qui décident de collaborer à l'initiative sans être rémunérés ${ }^{76}$. Quelles sont les raisons qui poussent ces acteurs à

74. Fondé en 2006, I'Arabic Network for Human Rights Information est une ONG égyptienne qui œuvre dans le domaine de la liberté d'expression et de publication. Voir http://anhri.net/

75. Entretien personnel, Le Caire, avril 2011.

76. Ce n'est qu'après quelques mois que son rédacteur-en-chef décide de vendre un terrain de propriété pour donner au projet un certain degré d'institutionnalisation et commencer à rétribuer une petite équipe de rédacteurs. 
participer à la création du site et à y travailler gratuitement ? Un article publié par Khaled Al-Balshy le 10 novembre sous le titre de " C'est pour cela qu'il était indispensable de revenir » donne quelques éléments de réponse à cette question :

" (...) Nous rêvions d'être présents en ce moment, de déclarer que nous refusons la tentative de falsifier la volonté du peuple... d'être une partie dans le jeu absurde de la transition du pouvoir en Egypte, ne serait-ce qu'avec nos mots après que le despotisme du pouvoir et la gaieté de l'opposition nous ont rendus inaptes à l'action et au changement de nos propres mains ... Nous nous consolons car nous sommes journalistes, notre parole est notre force et nos cœurs débordent d'amour pour les gens de ce peuple et de désir de changer un régime qui a dépassé la mesure, s'est montré récalcitrant, a tyrannisé et s'est enfoncé dans les profondeurs de sa corruption (...) c'est pour cela qu'il était indispensable de revenir à vous en ce moment. Notre retour à vous est notre tentative de sortir de la prison de la foi la plus faible vers la lande de l'action, même si cette action est faible et même si la diffusion de notre parole est limitée ... Nous parions sur vous lorsque nous sommes sortis parier sur notre amour pour vous et nous attendons votre aide, que vous soyez un soutien pour un groupe de jeunes qui ont voulu dire un mot de vérité en face d'un pouvoir injuste et d'un régime dont la corruption et l'oppression ont atteint un niveau jamais atteint dans le passé. Et en face de nouveaux Mamelouks qui nous ont ramenés au Moyen-âge et nous ont placés à la queue (du classement) des Nations. Peut être un seul regard à l'évolution de la nature et des mœurs des Egyptiens confirme-t-il la grandeur du crime qu'ils ont commis contre nous. Un régime qui a trouvé la manière de profiter de sa corruption en répandant la corruption parmi les citoyens de telle sorte que même lorsque chacun d'entre eux se replie sur soi-même et regarde les gros corrompus, il n'a pas la capacité d'agir ... Un régime qui a fait pression sur tous et les a écrasés, qui a orienté l'énergie de leur refus contre eux-mêmes et a fermé les portes de l'espoir en leur sein. (...) Pendant un an et demi, nous avons rêvé de vous et pour vous, pendant un an et demi nous avons désiré ardemment dire un mot de vérité, ce qui nous a été interdit pour des raisons économiques ou pour d'autres politiques, (nous avons désiré) terminer le chemin que nous avions commencé, c'est pour cela que nous revenons vers vous et nous sommes tous déterminés à continuer quels que soient les obstacles $(\ldots) »^{77}$.

77. "C'est pour cela qu'il était indispensable de revenir ", 10 novembre 2010 : http://elbadil.com/2010/11/10/28672/ 
Au-delà du lyrisme de ce qu'on pourrait définir en termes de "déclaration d'amour " aux lecteurs et au peuple égyptien dans son ensemble, ce texte donne plusieurs indications sur les contours du groupe d'acteurs impliqués dans la création du site web et sur les raisons qui les ont poussés à lancer le projet. Les artisans d'Al-BadîlAl-Jadîd y sont d'abord définis comme des "journalistes", puis comme un " groupe de jeunes qui ont voulu dire un mot de vérité en face d'un pouvoir injuste ... ». De manière générale, l'idée véhiculée par l'article d'Al-Balshy est que le lancement de la publication électronique soit le moyen, pour les jeunes reporters, de récupérer leur " identité » de journalistes dans un contexte où la presse est soumise à de puissantes contraintes économiques et politiques et où les professionnels de l'information ne sont pas libres d'exercer leur métier comme ils voudraient. Si l'expérience au quotidien Al-Badîl avait donné à ces jeunes l'opportunité de travailler à l'abri des contraintes de la censure et de l'autocensure, il s'agit de noter qu'après la clôture du journal plusieurs d'entre eux n'ont pas réussi à trouver un autre emploi, ou bien à s'habituer au climat de travail en vigueur au sein $\mathrm{d}^{\prime}$ autres institutions ${ }^{78}$. Dans un papier intitulé «A la mesure du rêve ", un autre journaliste d'Al-Badîl Al-Jadî̀, Mohamed El-Erian, confirme cette impression en disant : " L'expérience d'Al-Badîl était digne d'éloges, mais comme toutes les légendes elle a eu une fin tragique, et puisque nous sommes les " alternatifs » (al-badâyla) et nous rêvons et n'abandonnons pas le rêve, puisque nous croyons en le destin et avons découvert que les journaux ne nous ont pas séparés (...) nous avons décidé d'être, en dépit de ceux qui ne veulent pas que nous soyons ${ }^{79}$. Mais les propos de Khaled Al-Balshy montrent aussi que ces acteurs conçoivent leur profession non pas tant ou pas seulement comme un "gagne-pain ", mais comme une véritable mission. Etre journaliste signifie en effet « dire la vérité au pouvoir ", briser les tabous, dévoiler les méfaits ou les fautes du régime. Le journalisme est vécu à la fois comme un métier et comme un «devoir national indispensable ${ }^{80}$, vecteur d'expression $d^{\prime} u n$ engagement "total » contre le régime au pouvoir. Ce dernier semble véritablement être au centre des préoccupations des reporters d'Al-Badîl Al-Jadîd, comme le montrent les articles d'opinion qu'ils publient de temps en temps.

Produit d'un groupe de journalistes dont le dénominateur commun est l'opposition au régime et la volonté de le " démasquer », Al-Badîl Al-Jadîd est très différent de son prédécesseur. Certes, il maintient une

78. Certains d'entre eux ont d'ailleurs quitté le domaine pour exercer d'autres professions ou continuer leurs études.

79. Mohamed El-Erian, "A la mesure du rêve », 4 novembre 2010 : http:// elbadil.com/2010/11/04/30542/

80. Cfr. supra. 
ligne éditoriale " gauchiste ", car un grand nombre de ses rédacteurs, à commencer par Khaled Al-Balshy, revendiquent des orientations idéologiques situées à gauche. Dès sa parution, il traduit cette identité par des choix éditoriaux qui rappellent sous certains aspects ceux d'A/Badil. Le site se distingue ainsi par une rubrique intitulée "Société civile » où sont publiés des articles portant sur les différents mouvements contestataires, les ONGs, les protestations des travailleurs et des gens ordinaires. II fait montre d'un intérêt marqué pour la question des libertés publiques et la volonté de 'publiciser' tous les cas de torture et d'agressions commises par les forces de sécurité contre les citoyens égyptiens ainsi que d'informer les lecteurs sur la condition des détenus politiques. Cependant, il affirme son indépendance de tout courant ou parti politique et ambitionne d'être une plate-forme d'expression pour la gauche dans son ensemble. Ou encore, pour citer les mots de son rédacteur-en-chef, "un média exprimant des principes généraux de gauche, tels que celui de la justice sociale ou des libertés publiques ${ }^{81}$, mais qui s'éloigne des strictes questions doctrinales. Il est intéressant de remarquer que sa rubrique d'opinion intitulée "Hyde Park » est ouverte à "tous ceux qui veulent dire quelque chose de différent, soient-ils des gauchistes, des libéraux, ou des islamistes» ${ }^{82}$. Une étude de cette rubrique montre que les éditorialistes du site sont des figures de I'opposition tous courants confondus dont le point commun est de ne pas disposer de tribunes dans les autres médias traditionnels du pays. Ses collaborateurs les plus assidus sont à la fois des intellectuels, des activistes de la société civile, des blogueurs et des journalistes plus ou moins confirmés qui dans certains cas travaillent au sein d'institutions de presse étatiques mais qui ne peuvent pas, pour des raisons de type politique, s'exprimer dans leurs publications respectives.

Je voudrais conclure en faisant quelques considérations sur la façon dont Al-Badîl Al-Jadîd construit son rapport au mouvement révolutionnaire égyptien et la manière dont il s'engage dans la lutte symbolique pour la définition de la réalité que s'ouvre lors de la destitution du président Moubarak. Produit d'un groupe de jeunes unis par une même attitude vis-à-vis des autorités au pouvoir, il paraît somme toute logique qu'Al-Badîl Al-Jadîd se positionne très tôt et sans ambigüités du côté des appels à manifester le 25 janvier « contre la torture, la pauvreté et la corruption ${ }^{83}$. Ses journalistes publient eux-mêmes des messages adressés aux élites intellectuelles

81. Entretien personnel, Le Caire, avril 2011.

82. Ibid.

83. «Après le soulèvement tunisien... des activistes invitent à se révolter contre la torture, la pauvreté et la corruption devant le Ministère de l'intérieur le jour de la fête de la police », 15 janvier 2011 : http://elbadil. com/2011/01/15/9394/ 
de l'opposition et à toutes les forces nationales engagées dans la « lutte pour le changement " pour qu'elles descendent avec aux dans la rue ${ }^{84}$. Dès le 25 janvier, le site est en première ligne dans la couverture des protestations et des affrontements entre les manifestants et la police, ce qui lui vaudra quelques rétorsions de la part des autorités, dont une attaque à coup de bombes lacrymogènes le 5 février suite à laquelle ses reporters seront contraints de changer provisoirement de bureau.

Après la chute du président Moubarak, le site continue de se positionner clairement aux côtés du mouvement révolutionnaire et à contester la définition de la réalité promue par les nouvelles autorités au pouvoir. II suit de près l'affaire des "martyrs de la révolution » en publiant plusieurs interviews avec des membres des familles des victimes; il s'intéresse à la question des procès militaires dont sont victimes les activistes qui continuent à protester dans la rue et donne un large espace aux voix des groupes militants qui se battent contre les tribunaux spéciaux, notamment " Non aux procès militaires " $\left(L^{\prime}{ }^{\prime}\right.$ li-I-muhâkamât al-'askariya); il publie les appels à manifester lancés par les activistes tout au long de l'année 2011 et 2012, insistant sur la nécessité de poursuivre le chemin révolutionnaire. Aussi, dans une phase d'instabilité où les autorités militaires en charge du processus de transition semblent faire l'objet d'un véritable tabou, Al-Badîl Al-Jadîd est l'une des rares publications qui osent interroger, voire critiquer, les actions du Conseil suprême des forces armées ${ }^{85}$.

\section{CONCLUSION}

Cet article visait à retracer l'histoire d'une des publications privées dites indépendantes qui paraissent sur la scène médiatique égyptienne pendant les années 2000. Expérience singulière située à la croisée entre les univers du journalisme et du militantisme, elle n'en est pas moins représentative du contexte socio-historique plus large dans lequel elle émerge : celui d'un pays paralysé par le "blocage démocratique » et le fantôme de la transmission héréditaire du pouvoir, où la scène médiatique devient un lieu d'expression politique pour des acteurs en quête de nouveaux terrains d'engagement. Mais Al-Badîl participe aussi et surtout du processus de renouvellement générationnel à l'œuvre à la fois dans le champ journalistique et dans la société égyptienne dans

84. Khaled Al-Balshy, "Lettre au Dr. El-Baradei... et aux écrivains qui crient dans le désert... Soyez avec nous le 25 janvier», 19 janvier 2011 : http://elbadil.com/2011/01/19/19526/ ; Khalil Abou Chady, "Venez dire un mot le 25 janvier », 20 janvier 2011 : http://elbadil.com/2011/01/20/30747/ 85. Entretien avec Gamal Eid, directeur de I'ANRHI, Le Caire, mai 2011. 
son ensemble, qui entraîne avec lui l'émergence de nouvelles manières de concevoir la profession journalistique, d'une part, et l'engagement militant, de l'autre.

Il me reste à dire quelques mots sur la fin prématurée que connaît I'expérience d'Al-Badîl Al-Jadîd. Suite à la révolte janvier 2011, ses journalistes commencent à rechercher de nouveaux mécènes intéressés à financer leur publication électronique, l'idée étant de développer le site web mais aussi de travailler à la préparation d'un journal papier. A partir du mois de mai, ils reprennent contact avec deux des anciens propriétaires d'Al-Badîl (qui entre temps ont participé à la fondation $d^{\prime}$ un des partis de gauche émergeant sur la scène politique) et entament une nouvelle expérience de collaboration dans I'objectif de ressusciter le journal sous format hebdomadaire. Malgré l'enthousiasme initial et les espoirs suscités par cette "réconciliation », la publication ne verra jamais le jour. Au début de 2012, la licence de publication d'Al-Badîl est vendue à un nouveau groupe d'investisseurs qui paraissent avoir l'ambition à la fois de créer un grand portail électronique, de fonder un quotidien papier et de lancer une chaîne télévisuelle. Plus tard dans l'année, Khaled Al-Balshy est limogé de sa fonction de rédacteuren-chef du site à cause de divergences politiques avec les nouveaux propriétaires et plusieurs de ses collègues démissionnent en signe de solidarité avec lui. En octobre 2012, il lance une nouvelle publication électronique sous le nom emblématique de "Le Début. Pour une génération qui mérite de vivre » (Al-Bidâya. Li-jîl yastahiqqu al-hayât ${ }^{86}$. Début d'une nouvelle aventure journalistique et militante? Au-delà des détails, les dynamiques ayant conduit à la fin de l'expérience d'AlBadîl Al-Jadîd montrent que dans le contexte hyper-politisé et polarisé de I'après-25 janvier, où le secteur médiatique devient le site d'un investissement de plus en plus poussé de la part des élites économiques du pays, les journalistes demeurent soumis à des contraintes qui semblent à certains égards être encore plus lourdes qu'auparavant.

86. http://albedaiah.com/ 


\section{BIBLIOGRAPHIE}

Abdallah A., 1985, The Student Movement and National Politics in Egypt 1923-1973, Londres, Al-Saqi Books, 300 p.

Abdelfattah N., 2009, "L'éloge de l'extraordinaire, de ses créations et de ses vertus ", Swasiya, nº. 89/90, p. 5-6.

Al-anany K., 2010, "L'originalité de la pensée et l'ingéniosité de la méthode ", Rawaq Arabi, n. 53, p. 49-54.

Al-тонамy A., 2009, Al-ajiyâl fi-l-siyâsa al-masriya. Dirâsa hala li-jîl alsab'îniyât (Les générations et la politique en Egypte. Etude de cas de la génération 1970), Le Caire, Markaz al-Ahrâm li-I-dirâsat alsiyâsiya wa-l-istrâtîjiya, 260 p.

AmiR F. H., 2006, Al-sahâfa al-khâssa fi Misr (La presse privée en Egypte), Le Caire, Markaz al-hadâra al-'arabiya, 158 p.

BEN NEFISSA S., 2010, "Verrouillage autoritaire et mutation générale des rapports entre l'Etat et la société en Egypte», Confluences Méditerranée, nº. 75, p. 137-150.

BERNARD-MAUGIRON N., 2007, " Le printemps des juges et la réactualisation autoritaire en Égypte ", Politique africaine, $\mathrm{n}^{\circ}$. 108, p. 67-85.

Bourdieu P., 1984, "La jeunesse n'est qu'un mot », dans Questions de sociologie, Paris, Ed. Minuit, p. 143-154.

Braungart R. \& M., 1989, "Les générations politiques", dans Crête J. \& Favre P. (éds.), Générations et politique, Paris/Laval, EconomicaPUL, p. 7-52.

El-KhawaGa D., 1997, "Les droits de l'homme en Egypte. Dynamiques de relocalisation d'une référence occidentale », Egypte/Monde arabe, $\mathrm{n}^{\circ}$. 30-31, p. 231-250.

El-KHAWAGA D., 2003, «La génération seventies en Egypte. La société civile comme répertoire d'action alternative », dans BennaniChraibi M. et Fillieule O. (éds.), Résistances et protestations dans les sociétés musulmanes, Paris, Presses de Sciences Po, p. 270-292.

El-KhaWAGA D., 2003 (b), "La restructuration de I'audiovisuel en Égypte : I'imbrication de diverses logiques d'action », dans Mermier F. (éds), Mondialisation et nouveaux médias dans l'espace arabe, Paris, Maisonneuve \& Larose, p. 91-101.

GeRvasio G., 2010, Al-haraka al-mârkisîya fi Misr (1967-1981) (Le mouvement marxiste en Egypte (1967-1981)), Le Caire, Al-machrû' al-qawmî li-l-tarjâma, 451 p.

HamDY, N., 2002. "A Dream TV ComeTrue " Transnational Broadcasting Studies 8 (Spring/Summer). www.tbsjournal.com

HaSAN B., 2009, "Muhammad Al-Sayyid Sa'îd ra'id huqûq al-insân » (Mohammed Al-Sayyed Saïd le pionnier des droits de I'homme), Swasiya, ${ }^{\circ} .89 / 90$, p. 3.

HASSABO C., 2009, "Du rassemblement à l'effritement des Jeunes pour le changement égyptiens. L'expérience de "générations qui ont 
vécu et vivent toujours sous la loi d'urgence ", Revue Internationale de Politique Comparée, vol. 16, nº. 2, p. 241-261.

Kienle E., 2001, A Grand Delusion. Democracy and Economic Reform in Egypt, London, I. B. Tauris, 256 p.

Mannheim K., 1990, Le problème des générations, Paris, Nathan, 122 p. SHeHATA D., 2011, "Al-harakât al-chabâbiya wa thawrat 25 yanâyr » (Les mouvements de jeunes et la révolution du 25 janvier), Kurrâsât 'istrâtijiya, http://digital.ahram.org.eg/articles. aspx? Serial $=657099 \&$ eid $=6729$

ZAHRAN F., 2003, "Al-qam' al-fikrî dâkhil al-ahzâb al-siyâsiya fî Misr » (La repression intellectuelle au sein des partis politiques en Egypte), http://www.ahewar.org/debat/show.art.asp?aid=9728

ZAHRAN F., 2007, Al-harakât al-ijtimâ'iya al-jadîda (Les nouveaux mouvements sociaux), Cairo, Cairo Institute for Human Rights Studies, 66 p. 